\title{
A SARS-CoV-2 Infection High-Uptake Program on Healthcare Workers and Cancer Patients of the National Cancer Institute of Naples, Italy
}

\author{
Anna Crispo $^{1} \mathbb{D}$, Piergiacomo Di Gennaro ${ }^{1} \mathbb{D}$, Sergio Coluccia ${ }^{1}$, Sara Gandini ${ }^{2} \mathbb{D}$, Concetta Montagnese ${ }^{1, * \mathbb{D}}$, \\ Giuseppe Porciello ${ }^{1}{ }^{D}$, Flavia Nocerino ${ }^{1}$, Maria Grimaldi ${ }^{1}$, Mariangela Tafuri ${ }^{1}$, Assunta Luongo ${ }^{1}$, \\ Emanuela Rotondo ${ }^{1}$, Alfonso Amore ${ }^{3}$, Francesco Labonia ${ }^{4}$, Serena Meola 4 , Stefanie Marone ${ }^{4}$, Giovanni Pierro ${ }^{4}$, \\ Simona Menegozzo ${ }^{5}$, Leonardo Miscio ${ }^{5}$, Francesco Perri ${ }^{6}{ }^{\mathbb{D}}$, Maurizio Rainisio ${ }^{7} \mathbb{D}$, Attilio A. M. Bianchi ${ }^{8}$, \\ Ernesta Cavalcanti ${ }^{4}$, Marco Cascella ${ }^{9}$ (D) and Egidio Celentano ${ }^{1}$
}

check for updates

Citation: Crispo, A.; Di Gennaro, P.; Coluccia, S.; Gandini, S.; Montagnese, C.; Porciello, G.; Nocerino, F.; Grimaldi, M.; Tafuri, M.; Luongo, A.; et al. A SARS-CoV-2 Infection High-Uptake Program on Healthcare Workers and Cancer Patients of the National Cancer Institute of Naples, Italy. Healthcare 2022, 10, 205.

https://doi.org/10.3390/

healthcare10020205

Academic Editors: Christian Napoli and Abdel-Latif Mohamed

Received: 18 November 2021

Accepted: 16 January 2022

Published: 20 January 2022

Publisher's Note: MDPI stays neutral with regard to jurisdictional claims in published maps and institutional affiliations.

Copyright: (C) 2022 by the authors. Licensee MDPI, Basel, Switzerland. This article is an open access article distributed under the terms and conditions of the Creative Commons Attribution (CC BY) license (https:// creativecommons.org/licenses/by/ $4.0 /)$.
1 Epidemiology and Biostatistics Unit, Istituto Nazionale per lo Studio e la Cura dei Tumori, "Fondazione Pascale"_-IRCCS, 80131 Naples, Italy; a.crispo@istitutotumori.na.it (A.C.); piergiacomo.digennaro@istitutotumori.na.it (P.D.G.); sergio.coluccia@istitutotumori.na.it (S.C.); g.porciello@istitutotumori.na.it (G.P.); f.nocerino@istitutotumori.na.it (F.N.); m.grimaldi@istitutotumori.na.it (M.G.); mariangela.tafuri@istitutotumori.na.it (M.T.); assunta.luongo@istitutotumori.na.it (A.L.); e.rotondo@istitutotumori.na.it (E.R.); e.celentano@istitutotumori.na.it (E.C.)

2 Department of Experimental Oncology, European Institute of Oncology (IEO)_IRCCS, 20141 Milan, Italy; sara.gandini@ieo.it

3 Division of Surgery of Melanoma and Skin Cancer, Istituto Nazionale per lo Studio e la Cura dei Tumori, "Fondazione Pascale"_-IRCCS, 80131 Naples, Italy; a.amore@istitutotumori.na.it

4 Laboratory Medicine Unit, Istituto Nazionale per lo Studio e la Cura dei Tumori, "Fondazione Pascale" —IRCCS, 80131 Naples, Italy; francesco.labonia@istitutotumori.na.it (F.L.); serena.meola@istitutotumori.na.it (S.M.); stefanie.marone@istitutotumori.na.it (S.M.); giovanni.pierro@istitutotumori.na.it (G.P.); e.cavalcanti@istitutotumori.na.it (E.C.)

5 Medical Direction, Istituto Nazionale per lo Studio e la Cura dei Tumori, "Fondazione Pascale"—IRCCS, 80131 Naples, Italy; s.menegozzo@istitutotumori.na.it (S.M.); leonardo.miscio@istitutotumori.na.it (L.M.)

6 Head and Neck Medical and Experimental Oncology Unit, Istituto Nazionale per lo Studio e la Cura dei Tumori, "Fondazione Pascale"_-IRCCS, 80131 Naples, Italy; f.perri@istitutotumori.na.it

7 AbaNovus srl, 18038 Sanremo, Italy; Maurizio.Rainisio@AbaNovus.com

8 Directorate-General for Management, IRCCS Fondazione G. Pascale, 80131 Naples, Italy; a.bianchi@istitutotumori.na.it

9 Division of Anesthesia and Pain Medicine, Istituto Nazionale Tumori, IRCCS Fondazione G. Pascale, 80131 Naples, Italy; m.cascella@istitutotumori.na.it

* Correspondence: c.montagnese@istitutotumori.na.it; Tel.: +39-081-59031782 
the prevalence of patients tested varied from 100 (4.6\%) during the first wave to 168 (4.9\%) during the second wave $(p=0.8)$. The multivariate logistic analysis provided a significant $\mathrm{OR}$ for nurses $(\mathrm{OR}=2.24,95 \%$ CI 1.23-4.08, $p<0.001)$ compared to research, administrative staff, and other job titles. Conclusions: Our findings show that the positivity rate between the two waves in the HCWs increased over time but not in the CPs; therefore, the importance of adopting stringent measures to contain the shock wave of SARS-CoV-2 infection in the hospital setting was essential. Among HCWs, nurses are more exposed to contagion and patients who needed continuity in oncological care for diseases other than COVID-19, such as suspected cancer.

Keywords: SARS-CoV-2; COVID-19; healthcare workers; surveillance program; cancer patients

\section{Introduction}

Italy was the first European state to be seriously affected by the Severe acute respiratory syndrome coronavirus 2 (SARS-CoV-2) pandemic [1]. Remarkably, in the Italian peninsula, from the beginning of the pandemic to December 2020, 1,757,394 cases and 61,240 deaths were recorded; of which 167,433 cases and 2064 deaths occurred in the Campania region (South Italy) [1,2]. Thus, since the World Health Organization (WHO) declared Coronavirus Disease 19 (COVID-19) as a pandemic (11 March 2020), drastic preventive measures to contain the viral contagion, such as social distancing and confinement, and use of personal protective equipment were immediately adopted.

In the first phase of the pandemic, between February and June 2020, the epidemiological surveillance focused mainly on symptomatic or suspected cases or contacts with SARS-CoV-2 positives [3]. Later, in the second pandemic wave between September and December 2020, the epidemiological surveillance addressed different population groups including students, teachers, school workers, healthcare workers (HCWs), and patients who needed hospital treatment for diseases other than COVID-19, such as suspected cancer. This surveillance was achieved through serological tests and nasopharyngeal swabs. Among these categories, maximum attention was paid to HCWs since they can represent an important vehicle for the spread of the virus, especially among vulnerable populations, such as cancer patients who are at increased risk of becoming ill with more serious symptoms of SARS-CoV-2 infection [4-9]. In Italy, starting from March 2020, the National Cancer Institute of Naples (Istituto di Ricovero e Cura a Carattere Scientifico-IRCCS, Fondazione Pascale) promoted a surveillance program for HCWs and cancer patients admitted to our hospital [10].

A careful surveillance program that pays particular attention to both fragile patients and workers can result in different care realities. The aim is to structure a validated pathway that, through adaptations, can be useful in different contexts. In a particularly changing pandemic reality, the strengthening of preventive measures, associated with vaccination campaigns, remains the best strategy to safeguard health services already severely tested.

In this study, the results of this high-uptake program are presented.

\section{Materials and Methods}

\subsection{Study Design}

This is an observational prospective study aimed at describing the prevalence of SARSCoV-2 infection detected by molecular nasal and oropharyngeal swab RT-PCR testing in HCWs and cancer patients (CPs). These populations were followed from March 2020 to March 2021. According to the course of the SARS-CoV-2 epidemic in Italy [2,3], different periods (pandemic waves and transition phases) have been considered. In particular, the study period encompassed:

- The first wave, from 4 March 2020, to 31 May 2020;

- A transition phase, from 1 June 2020, to 27 September 2020;

- $\quad$ The second wave from 28 September 2020, to 3 January 2021; 
- $\quad$ The second transition phase from 4 January 2021, to 4 April 2021.

This study was approved by the Ethics Committee of the National Cancer Institute of Naples (IRCCS, Fondazione Pascale) (number 61/20).

\subsection{Sample}

This study involved all HCWs of the National Cancer Institute of Naples (IRCCS, Fondazione Pascale) who agreed to participate in the surveillance program (signed informed consent obtained) and all CPs who had to get a medical examination or had to be hospitalized in the Institute from 4 March 2020 to 4 April 2021.

For HCWs, the surveillance program included a periodic sampling through nasopharyngeal molecular swabs for SARS-CoV-2. The tests were performed in HCWs who were asymptomatic; symptomatic HCWs and those who refused to be tested were excluded. A subject was defined as symptomatic if any of the following symptoms/signs were manifested: fever, cough, dyspnea, chills, anosmia, ageusia.

SARS-CoV-2 positive HCWs did not have access to the hospital and were referred to the territorial healthcare system for testing and contact tracing. HCWs who were in contact with COVID-19 patients or SARS-CoV-2 infected individuals were included in a quarantine program by the territorial healthcare system. They were excluded from working activities until the favorable opinion of the health authorities.

According to our SARS-CoV-2 infection prevention policy, the pathways of CPs who had to be admitted to hospital for scheduled hospitalization, procedures or visits, had to carry out the molecular SARS-CoV-2 diagnostic test within $48 \mathrm{~h}$ before hospitalization. For each positive test, the local health authorities were alerted to manage the positive CPs and their contacts. They were referred to multispecialty centers for COVID-19 patients (Figure 1).

\subsection{Tests Used for SARS-CoV-2}

The Laboratory Medicine Unit of the National Cancer Institute of Naples (IRCCS, Fondazione Pascale) has been involved since the beginning of the program of health surveillance for all HCWs, as well as patients in the identification of SARS-CoV-2 infection. As recommended by the $\mathrm{WHO}$, molecular testing has been used as the reference method for the identification of SARS-CoV-2 infectious cases [11]. Nucleic acids extraction and subsequent Real-Time PCR (RT-PCR) detection of SARS-CoV-2 RNA from nasopharyngeal swabs were performed [12]. The Charité algorithm (Berlin, Germany) worked out by Corman et al. [13], which is based on RT-PCR SARS-CoV-2 detection of E and RdRp genes, was used as a reference method.

\subsection{Statistical Analysis}

The SARS-CoV-2 prevalence was calculated for each epidemic period (1st wave, 1st transition phase, 2nd wave, 2nd transition phase) and was reported according to the age, gender, department and job title of HCWs and the age, gender, type and number of accesses of patients; HCWs or CPs who had multiple tests in the same wave were taken into account and the repeated measures were excluded.

A univariate analysis was implemented for the 2nd wave only, according to HCWs' features and CPs through the Chi-Square test. Adjusted Odds Ratios (ORs) and 95\% Confidence Intervals (CIs) were estimated on the 2 nd wave by unconditional multiple logistic regression model with terms of age $(\leq 44,>44)$, gender, job title (ancillary services, non-medical-area, nurse, physician, research staff, administrative staff, technologist and other), department (clinical care, surgery, research, administrative and operational service), and molecular swabs $(1-3,4-5,6-11$, not present in table) to assess the main SARS-CoV-2 risk factors. In the second pandemic wave, a Cumulative Hazard Function by CPs' accesses and HCWs' job title was calculated; $p$-values were produced by the Log-rank test. Hazard Ratios and 95\% CIs were calculated through the Cox-model. The models were adjusted by 
age and gender. All analyses were performed using R software (version 4.0.2, R Core Team, Vienna, Austria).

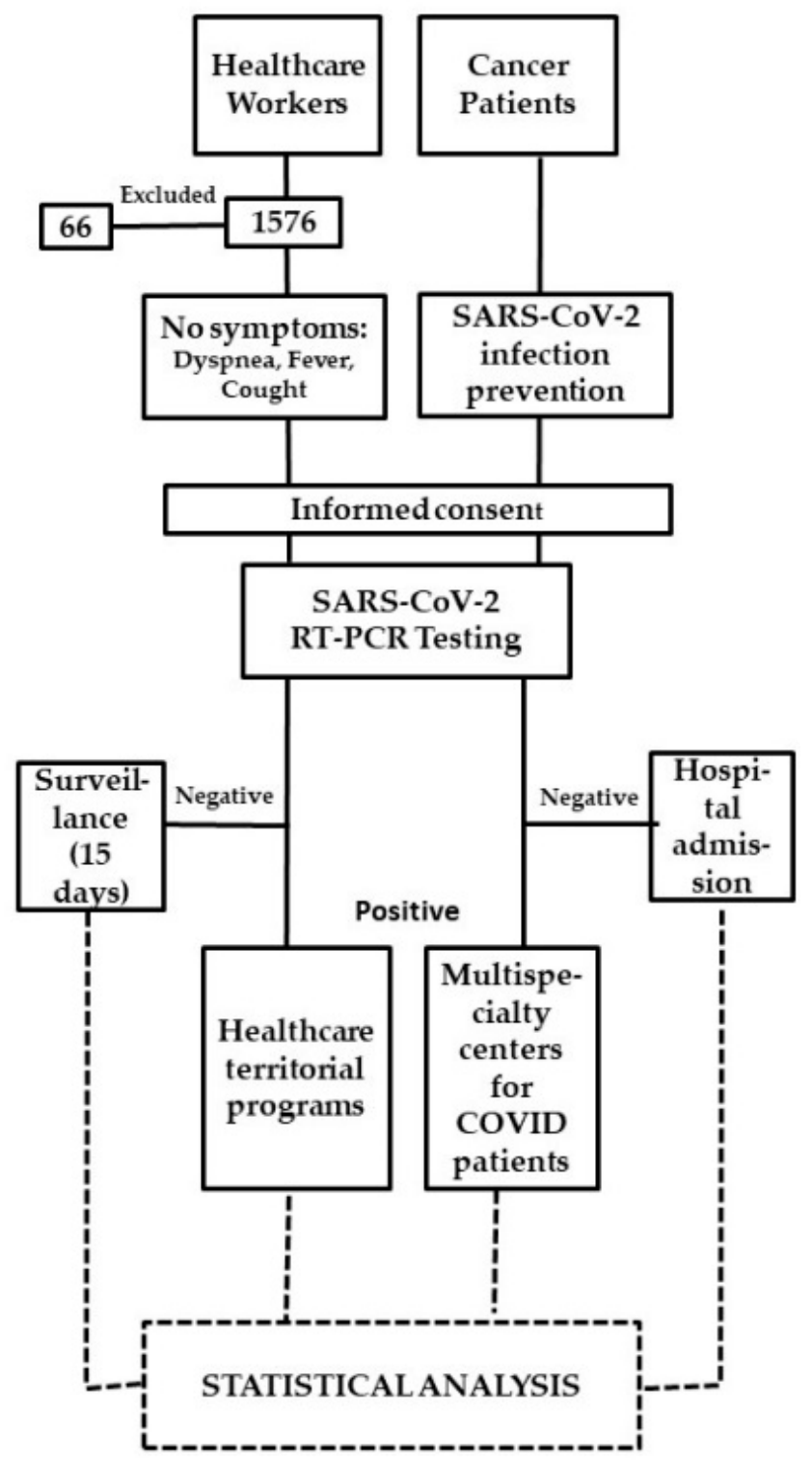

Figure 1. Study flow chart.

\section{Results}

A total of $1510 \mathrm{HCWs}$ were tested with molecular nasal and oropharyngeal swab RT-PCR testing for the detection of SARS-CoV-2 [10]. 1204 (79.7\%) and 1385 (91.7\%) of HCWs were tested during the first and the second waves, respectively. A total of 8733 CPs, who were admitted to the National Cancer Institute of Naples (IRCCS, Fondazione Pascale), were tested. A total of $2152(24.6 \%)$ and $3462(39.6 \%)$ of CPs were tested during the first and second waves, respectively. The total number of molecular swabs executed over the study period were 12,677 for HCWs and 15,155 for CPs. SARS-CoV-2 was detected in 20 (1.7\%) HCWs during the 1st wave, and $127(9.2 \%)$ in the second wave $(p<0.001)$; while among CPs, the prevalence varied from $100(4.6 \%)$ during the first wave to $168(4.9 \%)$ during the second wave $(p=0.8)$ (Table 1$)$. 
Table 1. SARS-CoV-2 incidence rate of Healthcare Workers (HCWs, Overall $N=5106$ swabs for 1510 healthcare workers, multiple tests in the same wave are excluded) and Cancer Patients (CPs; Overall $N=10,846$ accesses for 8733 patients, multiple accesses in same wave are excluded) from the first to the second wave of the pandemic.

\begin{tabular}{|c|c|c|c|c|c|c|c|c|c|c|c|c|}
\hline \multirow{2}{*}{ HCWs and CPs } & \multicolumn{3}{|c|}{$\begin{array}{c}\text { First Wave: } \\
\text { March-May } 2020\end{array}$} & \multicolumn{2}{|c|}{$\begin{array}{c}\text { 1^ Transition Phase } \\
\text { June-September } 2020\end{array}$} & & \multicolumn{2}{|c|}{$\begin{array}{c}\text { Second Wave } \\
\text { October-December } 2020\end{array}$} & \multicolumn{4}{|c|}{$\begin{array}{l}\text { 2^ Transition Phase } \\
\text { January-March } 2021\end{array}$} \\
\hline & HCWs & CPs & & HCWs & CPs & & HCWs & CPs & & HCWs & CPs & \\
\hline Total $(\%)^{1}$ & $\begin{array}{c}1204 \\
(79.7 \%)\end{array}$ & $\begin{array}{c}2152 \\
(24.6 \%)\end{array}$ & & $\begin{array}{c}1268 \\
(84.0 \%)\end{array}$ & $\begin{array}{c}1793 \\
(20.5 \%)\end{array}$ & & $\begin{array}{c}1385 \\
(91.7 \%)\end{array}$ & $\begin{array}{c}3462 \\
(39.6 \%)\end{array}$ & & $\begin{array}{c}1249 \\
(82.7 \%)\end{array}$ & $\begin{array}{c}3439 \\
(39.4 \%)\end{array}$ & \\
\hline \multirow[t]{2}{*}{ Positive (\%) } & $\begin{array}{c}20 \\
(1.7 \%)\end{array}$ & $\begin{array}{c}100 \\
(4.6 \%)\end{array}$ & & $\begin{array}{c}3 \\
(0.2 \%)\end{array}$ & $\begin{array}{c}14 \\
(0.8 \%)\end{array}$ & & $\begin{array}{c}127 \\
(9.2 \%)\end{array}$ & $\begin{array}{c}168 \\
(4.9 \%)\end{array}$ & & $\begin{array}{c}9 \\
(0.7 \%)\end{array}$ & $\begin{array}{c}40 \\
(1.2 \%)\end{array}$ & \\
\hline & \multicolumn{2}{|c|}{$\begin{array}{c}\text { Positive/TOT } \\
(\%)\end{array}$} & $p$-Value ${ }^{2}$ & \multicolumn{2}{|c|}{$\begin{array}{c}\text { Positive/TOT } \\
\text { (\%) }\end{array}$} & $p$-Value ${ }^{2}$ & \multicolumn{2}{|c|}{$\begin{array}{c}\text { Posivtive/TOT } \\
(\%)\end{array}$} & $p$-Value ${ }^{2}$ & \multicolumn{2}{|c|}{$\begin{array}{c}\text { Positive/TOT } \\
\text { (\%) }\end{array}$} & $p$-Value ${ }^{2}$ \\
\hline \multicolumn{13}{|l|}{ Age } \\
\hline$\leq 44$ & $\begin{array}{l}13 / 551 \\
(2.4 \%)\end{array}$ & $\begin{array}{l}8 / 269 \\
(3.0 \%)\end{array}$ & 0.77 & $\begin{array}{l}3 / 612 \\
(0.5 \%)\end{array}$ & $\begin{array}{l}5 / 261 \\
(1.9 \%)\end{array}$ & 0.10 & $\begin{array}{l}51 / 682 \\
(7.5 \%)\end{array}$ & $\begin{array}{c}28 / 530 \\
(5.3 \%)\end{array}$ & 0.16 & $\begin{array}{l}10 / 621 \\
(1.6 \%)\end{array}$ & $\begin{array}{l}10 / 615 \\
(1.6 \%)\end{array}$ & 1 \\
\hline$>44$ & $\begin{array}{l}7 / 653 \\
(1.1 \%) \\
\end{array}$ & $\begin{array}{c}92 / 1883 \\
(4.9 \%)\end{array}$ & $<0.001$ & $0 / 656$ & $\begin{array}{c}9 / 1532 \\
(0.6 \%) \\
\end{array}$ & 0.11 & $\begin{array}{l}76 / 703 \\
(10.8 \%) \\
\end{array}$ & $\begin{array}{c}140 / 2932 \\
(4.8 \%)\end{array}$ & $<0.001$ & $\begin{array}{l}2 / 625 \\
(0.3 \%) \\
\end{array}$ & $\begin{array}{c}30 / 2824 \\
(1.1 \%)\end{array}$ & 0.13 \\
\hline \multicolumn{13}{|l|}{ Gender } \\
\hline Male & $\begin{array}{l}6 / 588 \\
(1.0 \%)\end{array}$ & $\begin{array}{c}40 / 992 \\
(4.0 \%)\end{array}$ & 0.001 & $\begin{array}{l}1 / 566 \\
(0.2 \%)\end{array}$ & $\begin{array}{l}6 / 840 \\
(0.7 \%)\end{array}$ & 0.31 & $\begin{array}{l}66 / 638 \\
(10.3 \%)\end{array}$ & $\begin{array}{c}83 / 1683 \\
(4.9 \%)\end{array}$ & $<0.001$ & $\begin{array}{l}8 / 556 \\
(1.4 \%)\end{array}$ & $\begin{array}{c}24 / 1717 \\
(1.4 \%)\end{array}$ & 1 \\
\hline
\end{tabular}

${ }^{1}$ Total percentage represented the total number of HCWs and CPs included in the analysis. ${ }^{2}$ Pearson's Chi-square Test at significance level of $95 \%$. Bold numbers indicate statistically significant results. 
The highest prevalence among HCWs was detected for the second wave: 127 positives of 1385 HCWs $(9.2 \%)$ with $10.8 \%$ being over 44 years of age and $10.3 \%$ were male.

Amongst HCWs, Table 2 shows the distribution of positive to SARS-CoV-2 for Departments and Job Title. The highest incidence rate was observed for nurses (14.9\%).

Table 2. SARS-CoV-2 incidence rate of Healthcare Workers (HCWs; $N=1510$ ) from the first to the second wave of the pandemic.

\begin{tabular}{|c|c|c|c|c|}
\hline $\begin{array}{l}\text { Healthcare Workers } \\
\text { (HCWs) }\end{array}$ & $\begin{array}{c}\text { First Wave } \\
\text { March-May } 2020\end{array}$ & $\begin{array}{c}1^{\wedge} \text { Transition Phase } \\
\text { June-September } 2020^{2}\end{array}$ & $\begin{array}{c}\text { Second Wave } \\
\text { October-December } 2020^{3}\end{array}$ & $\begin{array}{l}2^{\wedge} \text { Transition Phase } \\
\text { January-March } 2021^{4}\end{array}$ \\
\hline \multicolumn{5}{|l|}{ Departments } \\
\hline Clinical care & $8 / 403(2.0 \%)$ & $0 / 432$ & $46 / 444(10.4 \%)$ & $2 / 418(0.5 \%)$ \\
\hline Surgery & $11 / 293(3.8 \%)$ & $1 / 315(0.3 \%)$ & $34 / 332(10.2 \%)$ & $4 / 305(1.3 \%)$ \\
\hline Research & $0 / 206$ & $1 / 240(0.4 \%)$ & $16 / 248(6.5 \%)$ & $2 / 234(0.9 \%)$ \\
\hline Administrative & $0 / 150$ & $1 / 175(0.6 \%)$ & $14 / 203(6.9 \%)$ & $0 / 183$ \\
\hline Operational Services & $1 / 152(0.7 \%)$ & $0 / 105$ & $17 / 156(10.9 \%)$ & $1 / 105(1.0 \%)$ \\
\hline \multicolumn{5}{|l|}{ Job Title } \\
\hline Ancillary services ${ }^{1}$ & $1 / 152(0.7 \%)$ & $0 / 105$ & $17 / 156(10.9 \%)$ & $1 / 105(1.0 \%)$ \\
\hline Non medical-area & $1 / 173(0.6 \%)$ & 0/185 & $13 / 193(6.7 \%)$ & $2 / 189(1.1 \%)$ \\
\hline Nurse & $4 / 356(1.1 \%)$ & $1 / 387(0.3 \%)$ & $62 / 416(14.9 \%)$ & 4/377 (1.1\%) \\
\hline Physician & $7 / 238(2.9 \%)$ & $1 / 247(0.4 \%)$ & $12 / 251(4.8 \%)$ & $0 / 233$ \\
\hline Research staff & $7 / 205(3.4 \%)$ & $0 / 242$ & $19 / 252(7.5 \%)$ & $2 / 236(0.8 \%)$ \\
\hline $\begin{array}{l}\text { Techno/Administr. } \\
\text { Staff/Other }\end{array}$ & $0 / 80$ & $1 / 101(1.0 \%)$ & $4 / 114(3.5 \%)$ & $0 / 105$ \\
\hline \multicolumn{5}{|l|}{ Molecular Swabs } \\
\hline Total (\%) & $1877(14.8 \%)$ & 2374 (18.7\%) & $5951(46.9 \%)$ & 2475 (19.5\%) \\
\hline Mean (SD) & $1.56(1.09)$ & $1.87(0.85)$ & $4.30(1.70)$ & $1.98(1.28)$ \\
\hline
\end{tabular}

Among $\mathrm{CPs}$, the prevalence during the first wave was similar to that observed in the second (4.6\% and $4.9 \%$, respectively); the number of positive patients increased with the number of accesses during the second wave (2.1\% one access $17.7 \%>4$ accesses) (Table 3$)$.

Table 3. SARS-CoV-2 incidence rate for Cancer Patients (CPs; $N=8733)$ from the first to second wave of the pandemic.

\begin{tabular}{ccccc}
\hline Cancer Patients & $\begin{array}{c}\text { First Wave: } \\
\text { March-May 2020 }\end{array}$ & $\begin{array}{c}\text { 1^ Transition Phase } \\
\text { June-September 2020 }\end{array}$ & $\begin{array}{c}\text { Second Wave } \\
\text { October-December 2020 }\end{array}$ & $\begin{array}{c}\text { 2` Transition Phase }_{\text {January-March 2021 }} \\
\text { January }\end{array}$ \\
\hline Number of accesses & & & & \\
One & $56 / 1562(3.6 \%)$ & $7 / 1338(0.5 \%)$ & $50 / 2349(2.1 \%)$ & $22 / 2741(0.8 \%)$ \\
Two & $33 / 494(6.7 \%)$ & $3 / 344(0.9 \%)$ & $57 / 757(7.5 \%)$ & $10 / 500(2.0 \%)$ \\
Three & $8 / 78(10.3 \%)$ & $2 / 76(2.6 \%)$ & $35 / 209(16.7 \%)$ & $3 / 102(2.9 \%)$ \\
Four or more & $3 / 18(16.7 \%)$ & $2 / 35(5.7 \%)$ & $26 / 147(17.7 \%)$ & $5 / 96(5.2 \%)$ \\
\hline Molecular Swabs & & & & $4714(31.1 \%)$ \\
Total (\%) & $2859(18.9 \%)$ & $2405(15.9 \%)$ & $5177(34.2 \%)$ & $1.37(1.21)$ \\
Mean for patient (SD) & $1.33(0.59)$ & $1.34(0.68)$ & $1.50(0.92)$ & \\
\hline
\end{tabular}

$\mathrm{SD}$, standard deviation.

Table 4 shows the associations between SARS-CoV-2 positive HCWs and features during the second wave. The univariate analysis showed that there were significant associations between virus positivity and age and job title. Notably, a statistically significant risk to be infected (OR $=2.2495 \%$ CI 1.23-4.08) was observed in nurses compared to research/administrative staff. 
Table 4. Univariate and multivariate analysis in the second pandemic wave for HCWs (Healthcare Workers).

\begin{tabular}{|c|c|c|c|c|c|c|}
\hline \multirow{3}{*}{ Healthcare Workers } & \multicolumn{6}{|c|}{$\begin{array}{c}\text { Second Wave } \\
\text { October-December } 2020^{1}\end{array}$} \\
\hline & \multicolumn{4}{|c|}{ Univariate } & \multicolumn{2}{|c|}{ Multivariate } \\
\hline & Negative & Positive (\%) & $p$-Value ${ }^{2}$ & Effect-Size $^{4}$ & OR $(95 \%$ CI) & $p$-Value ${ }^{3}$ \\
\hline Age & & & 0.04 & 0.06 & & 0.06 \\
\hline$\leq 44$ & 631 & $51(7.5 \%)$ & & & $1+$ & \\
\hline$>44$ & 627 & $76(10.8 \%)$ & & & $1.44(0.98-2.12)$ & \\
\hline Gender & & & 0.6 & 0.01 & & 0.2 \\
\hline Male & 616 & $66(9.7 \%)$ & & & $1+$ & \\
\hline Female & 642 & $61(8.7 \%)$ & & & $0.80(0.55-1.17)$ & \\
\hline Job Title & & & $<0.001$ & 0.07 & & $<0.001$ \\
\hline Research, administrative staff and other & 343 & $23(6.3 \%)$ & & & $1 \dagger$ & \\
\hline Ancillary services & 139 & $17(10.9 \%)$ & & & $1.68(0.55-1.17)$ & \\
\hline Non medical-area & 180 & $13(6.7 \%)$ & & & $0.96(0.47-1.98)$ & \\
\hline Nurse & 354 & $62(14.9 \%)$ & & & $2.24(1.23-4.08)$ & \\
\hline Physician & 239 & $12(4.8 \%)$ & & & $0.58(0.26-1.27)$ & \\
\hline Departments & & & 0.3 & 0.03 & & 0.6 \\
\hline Research, administrative and operational services & 560 & $47(7.7 \%)$ & & & $1+$ & \\
\hline Clinical care & 398 & $46(10.4 \%)$ & & & $1.24(0.71-2.17)$ & \\
\hline Surgery & 298 & $34(10.2 \%)$ & & & $1.02(0.54-1.91)$ & \\
\hline
\end{tabular}

Table 5 illustrates the associations between SARS-CoV-2 positive CPs and features during the second wave.

Table 5. Univariate and Multivariate analysis in the Second pandemic wave for CPs.

\begin{tabular}{|c|c|c|c|c|c|c|}
\hline \multirow{3}{*}{ Cancer Patients (CPs) } & \multicolumn{6}{|c|}{$\begin{array}{c}\text { Second Wave } \\
\text { October-December } 2020\end{array}$} \\
\hline & \multicolumn{4}{|c|}{ Univariate } & \multicolumn{2}{|c|}{ Multivariate } \\
\hline & Negative & Positive (\%) & $p$-Value ${ }^{1}$ & Effect-Size $^{3}$ & OR $(95 \%$ CI $)$ & $p$-Value ${ }^{2}$ \\
\hline Age & & & 0.7 & 0.06 & & 0.39 \\
\hline$\leq 44$ & 502 & $28(5.3 \%)$ & & & $1+$ & \\
\hline$>44$ & 2792 & $140(4.8 \%)$ & & & $0.83(0.54-1.27)$ & \\
\hline Gender & & & 0.9 & 0.01 & & 0.57 \\
\hline Male & 1600 & $83(4.9 \%)$ & & & $1+$ & \\
\hline Female & 1694 & $85(4.8 \%)$ & & & $1.10(0.80-1.51)$ & \\
\hline Number of accesses & & & $<0.001$ & 0.1 & & $<0.001$ \\
\hline One & 2299 & $50(2.1 \%)$ & & & $1+$ & \\
\hline Two & 700 & $57(7.5 \%)$ & & & $3.76(2.55-5.55)$ & \\
\hline Three & 174 & $35(16.7 \%)$ & & & $9.38(5.92-14.86)$ & \\
\hline Four or more & 121 & $26(17.7 \%)$ & & & $10.12(6.07-16.86)$ & \\
\hline
\end{tabular}

${ }^{1} p$-value referred to a Pearson's Chi-square test, significance level evaluated at $5 \% .{ }^{2}$ Logistic regression model adjusted for age, gender, number of accesses. ${ }^{3}$ Cramer's V statistic. Bold numbers indicate statistically significant results. + indicate reference category.

The univariate analysis reports that there was a significant association with the number of accesses. This finding was confirmed in the multivariate analysis where the risk categories: two, three and four or more accesses were statistically significant compared to one access (ORs $=3.76,9.38$, and 10.12, respectively).

Figure 2 shows the statistically significant infection cumulative hazard function either for CPs' access (A) $p<0.001$ or HCWs' job title (B) $p<0.001$. 


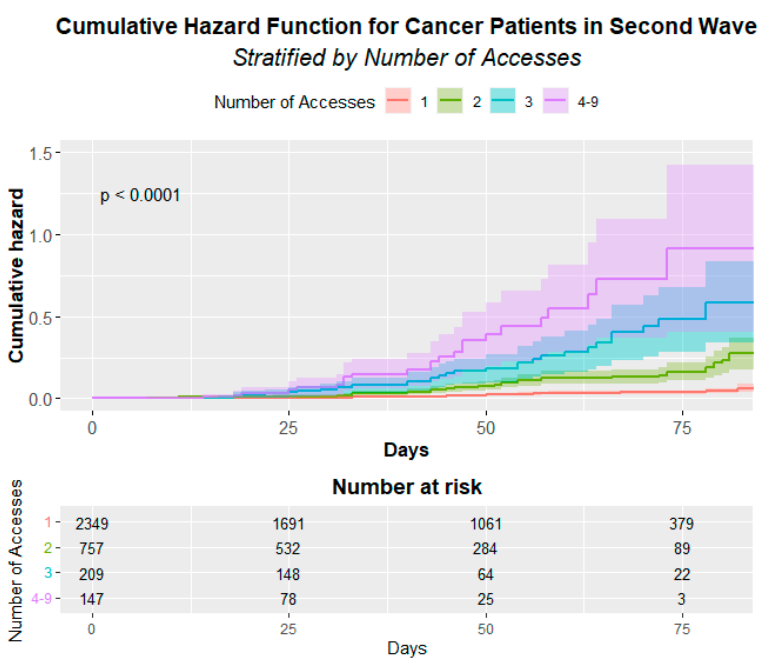

(a)

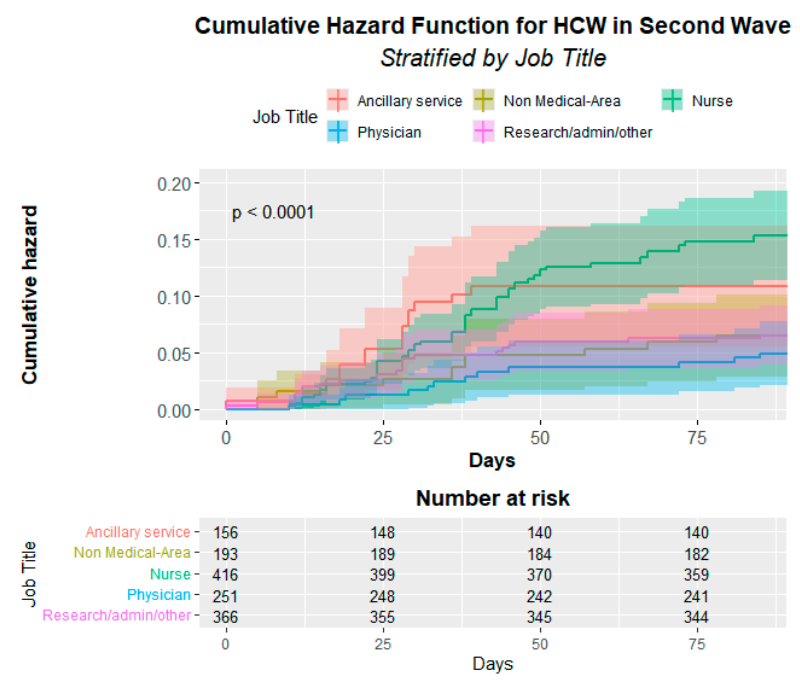

(b)

Figure 2. Cumulative Hazard Function for cancer patients (a) and Healthcare Workers (b). (a) Infection Cumulative Hazard Function by patient's accesses in the second pandemic wave. The $p$-value of the Log-rank test is at the top left. (b) Infection Cumulative Hazard Function by HCWs' job title (Healthcare Workers' Job Title) in the second pandemic wave. The $p$-value of the Log-rank test is at the top left.

The Cox multivariate analysis demonstrates a significant risk for CPs' access to be infected that increase from 4.13 for two access to 21.04 for four access and more (see Table 6).

Table 6. Cox-model for CPs in the second pandemic wave.

\begin{tabular}{ccc}
\hline $\begin{array}{c}\text { Cox-Model for } \\
\text { Cancer Patients (CPs) }\end{array}$ & \multicolumn{2}{c}{$\begin{array}{c}\text { Second Wave } \\
\text { October-December } 2020\end{array}$} \\
\hline & $\begin{array}{c}\text { HR }{ }^{1} \\
(95 \% \mathrm{CI})\end{array}$ & $p$-Value \\
\hline Number of accesses & $1+$ & \\
One & $4.13(2.82-6.04)$ & $<0.001$ \\
Two & $10.80(6.99-16.68)$ & $<0.001$ \\
Three & $21.04(13.09-33.82)$ & $<0.001$ \\
Four or more & & \\
\hline${ }^{1}$ Adjusted by age and gender. Bold numbers indicate statistically significant results. + indicate reference category.
\end{tabular}

The Cox multivariate analysis also confirms the significant risk for nurses $(\mathrm{HR}=2.05$ 95\% CI 1.16-3.64) (Table 7).

Table 7. Cox-model for HCWs (Healthcare Workers) in the Second pandemic wave.

\begin{tabular}{|c|c|c|}
\hline \multirow[t]{2}{*}{$\begin{array}{c}\text { Cox-Model for } \\
\text { Healthcare Workers (HCWs) }\end{array}$} & \multicolumn{2}{|c|}{$\begin{array}{c}\text { Second Wave } \\
\text { October-December } 2020\end{array}$} \\
\hline & $\begin{array}{c}\text { HR }^{1} \\
(95 \% \mathrm{CI})\end{array}$ & $p$-Value \\
\hline Job Title & & \\
\hline Research and admin, staff/other & $1+$ & \\
\hline Ancillary services & $1.70(0.88-3.29)$ & 0.117 \\
\hline Non medical-area & $0.95(0.48-1.91)$ & 0.895 \\
\hline Nurse & $2.05(1.16-3.64)$ & 0.014 \\
\hline Physician & $0.60(0.28-1.29)$ & 0.189 \\
\hline
\end{tabular}

${ }^{1}$ Adjusted by age, gender and Department. Bold numbers indicate statistically significant results. + indicate reference category. 


\section{Discussion}

Health surveillance on HCWs and patients represents a key strategy to strengthen and protect a workforce and the weakest and most compromised individuals, such as hospitalized patients [14]. Once transmission between asymptomatic subjects was known [15], periodic health surveillance among asymptomatic or mild-symptomatic HCWs helped to reduce the risk of infection to colleagues, their families, and patients in the workplace $[14,16]$. During the first phase of the SARS-CoV-2 pandemic, several guidelines were developed by the WHO, the European Centre for Disease Prevention and Control (ECDC), and the European Agency for Safety and Health at Work (EU-OSHA) to reduce SARS-CoV-2 risk of infection in HCWs; however, the HCWs could themselves become part of risk reduction strategies, through direct participation in all activities related to risk mitigation, monitoring and facilitating the reintegration of other workers previously affected by SARS-CoV-2. Furthermore, the HCWs involvement cannot be separated from the organization of "Health surveillance" with the purpose of planning adequate protection measures for fragile categories of workers [17]. Hospitalized cancer patients require ongoing evaluation and immediate treatment even during a pandemic. Nevertheless, since these patients are primarily immunocompromised, they are at greater risk of serious COVID-19 [18].

Our work shows the results of a surveillance program on HCWs and patients of an oncological facility in Campania (Italy). Notably, a higher incidence of infections among HCWs than in the general population was observed [19]. This finding confirmed the results of a previous prospective study that demonstrated an increased SARS-CoV-2 infection prevalence among HCWs (7.3\%) compared to non-HCWs (0.4\%) [20]. Several studies reported a considerable risk of contracting SARS-CoV-2 infection among HCWs [19-23], even if the incidence of severe disease and deaths was significantly low [24].

To plan a safe working environment and to maintain effective healthcare services, many studies focused on the risks associated with specific roles [25,26]. Investigations have suggested that HCWs at increased risk include porters, cleaners, healthcare-assistants, therapists, junior clinicians, but mostly nurses $[27,28]$. In line with the results of other studies, in our investigation, nurses were the largest group among HCWs to be infected. These HCWs are more prone to care for patients with suspected or confirmed COVID19, mainly in the hospital with a higher proportion of patients with COVID-19 [20]. In addition to the high risk of SARS-CoV-2 infection for healthcare professionals, it has been seen that they have experienced several mental problems, such as anxiety, stress, and burnout syndrome, a further problem to the already complicated working conditions in the pandemic phase. This could suggest the design of ad hoc programs for the prevention of mental disorders alongside health surveillance [29-33]. In support of this, it was shown that work overload and stress represented risk factors in contracting infectious diseases; in particular, this risk increased as the number of working hours increased, exposing HCWs for a longer time to patients, viruses, and mental stress [34].

Our findings show that the positivity rate between the two waves in the HCWs increased over time but not in the CPs. Therefore, the importance of adopting stringent measures to contain the shock wave of SARS-CoV-2 infection in the hospital setting was essential. Among HCWs, nurses are more exposed to contagion and patients who needed continuity in oncological care for diseases other than COVID-19, such as suspected cancer.

Notably, for CPs, the risk of infection increases with the number of accesses. It is necessary, therefore, to structure safe care pathways and organize, where possible, ad hoc methods for the provision of care. Our findings, for example, support a telemedicine-based approach for frail patients. This strategy represents a great opportunity to facilitate continued assistance for patients improving their access to care. Furthermore, telemedicine is cost-effective and can facilitate patient-centered treatments [35]. At the Cancer Institute, this model has been provided since the first COVID-19 wave [9] and will also be implemented in the post-pandemic era [36].

The major limitation of our study is that it was conducted in the pre-vaccinal era; however, the data that emerged suggest the importance of information and education 
programs [37]. Another limitation is that it is a single-center analysis: consequently, results may not be applicable to different healthcare settings. Nevertheless, since the beginning of the pandemic, our cancer center was declared 'COVID-free', and diagnosed or suspected COVID-19 patients were not admitted. In particular, CPs who were SARS-CoV-2 positive or symptomatic COVID-19 patients were referred to multispecialty centers for COVID-19 patients. Thus, all HCWs had the same risk of becoming infected in the hospital setting. Presumably, the nurses contracted the infection in an out-of-hospital setting. Indeed, during the most hectic phases of the pandemic, many nurses were recruited for community assistance programs.

\section{Conclusions}

Our findings underline that the adoption of stringent measures has been essential to contain the shock wave of SARS-CoV-2 infection in the hospital setting. In a far-sighted way, these measures involved both staff and patients.

We found that the positivity rate between the two waves in the HCWs increased over time, but not in the CPs. Additionally, our results suggest the adoption of safety measures in the HCWs, even if this is not directly related to the positivity of the CPs.

Among HCWs, nurses are at greater risk than administrative staff. Our findings clearly recommend the constant use of individual protection devices for nurses and that they undergo COVID-19 surveillance, particularly more frequently for those who participate in community assistance programs.

Author Contributions: Conceptualization, A.C., S.G., M.R., M.C. and E.C. (Egidio Celentano); methodology, A.C., P.D.G., S.C. and M.T.; software, P.D.G., S.C. and M.T.; validation, A.C., P.D.G., S.C., S.G., C.M., G.P. (Giuseppe Porciello), F.N., M.G., M.T., A.L., E.R., A.A., F.L., S.M. (Serena Meola), S.M. (Stefanie Marone), G.P. (Giovanni Pierro), S.M. (Simona Menegozzo), L.M., F.P., M.R., E.C. (Ernesta Cavalcanti), M.C., E.C. (Egidio Celentano); formal analysis, P.D.G., S.C. and M.T.; investigation, F.L., S.M. (Serena Meola), S.M. (Stefanie Marone), G.P. (Giovanni Pierro); resources, L.M., S.M. (Simona Menegozzo), E.C. (Ernesta Cavalcanti) and E.C. (Egidio Celentano); data curation, A.C., P.D.G., S.C., M.T., S.M. (Simona Menegozzo), C.M., G.P. (Giuseppe Porciello), F.N., M.G., A.L. and E.R.; writing-original draft preparation, A.C., C.M., G.P. (Giuseppe Porciello), and E.C. (Egidio Celentano); writing-review and editing, A.C., P.D.G., S.C., S.G., C.M., G.P. (Giuseppe Porciello), F.N., M.G., M.T., A.L., E.R., A.A., F.L., S.M. (Serena Meola), S.M. (Stefanie Marone), G.P. (Giovanni Pierro), S.M. (Simona Menegozzo), L.M., F.P., M.R., A.A.M.B., E.C. (Ernesta Cavalcanti), M.C. and E.C. (Egidio Celentano); visualization, A.C., P.D.G., S.C. and M.T.; supervision, A.C., S.G., C.M., G.P. (Giuseppe Porciello), F.N., M.G., A.L., E.R., A.A., F.L., S.M. (Serena Meola), S.M. (Stefanie Marone), G.P. (Giovanni Pierro), S.M. (Simona Menegozzo), L.M., F.P., M.R., E.C., M.C., E.C. (Egidio Celentano); project administration, L.M., A.A.M.B., S.M. (Simona Menegozzo) and E.C. (Ernesta Cavalcanti); funding acquisition, L.M., E.C. (Ernesta Cavalcanti) and E.C. (Egidio Celentano). All authors have read and agreed to the published version of the manuscript.

Funding: This research received no external funding.

Institutional Review Board Statement: The study was conducted according to the guidelines of the Declaration of Helsinki, and approved by the Ethics Committee of the National Cancer Institute of Naples (IRCCS, Fondazione Pascale) (protocol code 61/20, 20/01/2021).

Informed Consent Statement: Informed consent was obtained from all subjects involved in the study.

Data Availability Statement: The data presented in this study are openly available in Zenodo at https: / / doi.org/10.5281/zenodo.5582606 (accessed on 17 November 2021).

Conflicts of Interest: The authors declare no conflict of interest. 


\section{References}

1. Gagliano, A.; Villani, P.G.; Co', F.M.; Manelli, A.; Paglia, S.; Bisagni, P.A.G.; Perotti, G.; Storti, E.; Lombardo, M. COVID-19 Epidemic in the Middle Province of Northern Italy: Impact, Logistics, and Strategy in the First Line Hospital. Disaster Med. Public Health Prep. 2020, 14, 372-376. [CrossRef]

2. COVID-19 Situazione Italia—Dati del Dipartimento della Protezione Civile 2021. Available online: https://www.salute.gov.it/po rtale/nuovocoronavirus / dettaglioContenutiNuovoCoronavirus.jsp?area=nuovoCoronavirus\&id=5351\&lingua=italiano\&me nu=vuoto (accessed on 3 May 2021).

3. Italian Ministry of Health. Coronavirus. Available online: https://www.salute.gov.it/portale/nuovocoronavirus/dettaglioCont enutiNuovoCoronavirus.jsp?lingua=italiano\&id=5373\&area=nuovoCoronavirus\&menu=vuoto (accessed on 3 May 2021).

4. $\quad$ Bielicki, J.A.; Duval, X.; Gobat, N.; Goossens, H.; Koopmans, M.; Tacconelli, E.; van der Werf, S. Monitoring approaches for health-care workers during the COVID-19 pandemic. Lancet Infect. Dis. 2020, 20, e261-e267. [CrossRef]

5. Moscola, J.; Sembajwe, G.; Jarrett, M.; Farber, B.; Chang, T.; McGinn, T.; Davidson, K.W. Northwell Health COVID-19 Research Consortium. Prevalence of SARS-CoV-2 Antibodies in Health Care Personnel in the New York City Area. JAMA 2020, 324, 893-895. [CrossRef]

6. Liu, C.; Zhao, Y.; Okwan-Duodu, E.A.D.; Basho, R.; Cui, X. COVID-19 in cancer patients: Risk, clinical features, and management. Cancer Biol. Med. 2020, 17, 519-527. [CrossRef]

7. Lee, K.A.; Ma, W.; Sikavi, D.R.; Drew, D.A.; Nguyen, L.H.; Bowyer, R.C.E.; Cardoso, M.J.; Fall, T.; Freidin, M.B.; Gomez, M.; et al. Cancer and Risk of COVID-19 Through a General Community Survey. Oncologist 2020, 26, e182-e185. [CrossRef]

8. Iorio, G.C.; Ammendolia, A.; Marotta, N.; Ricardi, U.; de Sire, A. A bond between rheumatic diseases and cancer in the elderly: The interleukin-6 pathway. Int. J. Rheum. Dis. 2021, 24, 1317-1320. [CrossRef]

9. Crispo, A.; Montagnese, C.; Perri, F.; Grimaldi, M.; Bimonte, S.; Augustin, L.S.; Amore, A.; Celentano, E.; Di Napoli, M.; Cascella, M.; et al. COVID-19 Emergency and Post-Emergency in Italian Cancer Patients: How Can Patients Be Assisted? Front. Oncol. 2020, 10, 1571. [CrossRef]

10. Buonaguro, F.M.; Botti, G.; Ascierto, P.A.; Pignata, S.; Ionna, F.; Delrio, P.; Petrillo, A.; Cavalcanti, E.; Di Bonito, M.; Perdonà, S.; et al. The clinical and translational research activities at the INT_IRCCS "Fondazione Pascale" cancer center (Naples, Italy) during the COVID-19 pandemic. Infect. Agents Cancer 2020, 15, 69. [CrossRef]

11. Laboratory testing strategy recommendations for COVID-19-Interim guidance 2020. Available online: https://apps.who.int/iris/bi tstream/handle/10665/331509/WHO-COVID-19-lab_testing-2020.1-eng.pdf?sequence=1\&isAllowed=y (accessed on 3 May 2021).

12. Jin, Y.-H.; Cai, L.; Cheng, Z.-S.; Cheng, H.; Deng, T.; Fan, Y.-P.; Fang, C.; Huang, D.; Huang, L.-Q.; Huang, Q.; et al. A rapid advice guideline for the diagnosis and treatment of 2019 novel coronavirus (2019-nCoV) infected pneumonia (standard version). Mil. Med. Res. 2020, 7, 4. [CrossRef]

13. Corman, V.M.; Landt, O.; Kaiser, M.; Molenkamp, R.; Meijer, A.; Chu, D.K.; Bleicker, T.; Brünink, S.; Schneider, J.; Schmidt, M.L.; et al. Detection of 2019 novel coronavirus (2019-nCoV) by real-time RT-PCR. Eurosurveillance 2020, 25, 2000045. [CrossRef]

14. Black, J.R.M.; Bailey, C.; Przewrocka, J.; Dijkstra, K.K.; Swanton, C. COVID-19: The case for health-care worker screening to prevent hospital transmission. Lancet 2020, 395, 1418-1420. [CrossRef]

15. Huff, H.V.; Singh, A. Asymptomatic Transmission During the Coronavirus Disease 2019 Pandemic and Implications for Public Health Strategies. Clin. Infect. Dis. 2020, 71, 2752-2756. [CrossRef]

16. Barranco, R.; Ventura, F. COVID-19 and infections in health care workers: An emerging problem. Med.-Leg. J. 2020, 88, 65-66. [CrossRef]

17. Iavicoli, S.; Boccuni, F.; Buresti, G.; Gagliardi, D.; Persechino, B.; Valenti, A.; Rondinone, B.M. Risk assessment at work and prevention strategies on COVID-19 in Italy. PLoS ONE 2021, 16, e0248874. [CrossRef]

18. Gosain, R.; Abdou, Y.; Singh, A.; Rana, N.; Puzanov, I.; Ernstoff, M.S. COVID-19 and Cancer: A Comprehensive Review. Curr. Oncol. Rep. 2020, 22, 53. [CrossRef]

19. Nguyen, L.H.; Drew, D.A.; Joshi, A.D.; Guo, C.G.; Ma, W.; Mehta, R.S.; Sikavi, D.R.; Lo, C.H.; Kwon, S.; Song, M.; et al. Risk of COVID-19 among frontline healthcare workers and the general community: A prospective cohort study. medRxiv 2020. [CrossRef]

20. Barrett, E.S.; Horton, D.B.; Roy, J.; Gennaro, M.L.; Brooks, A.; Tischfield, J.; Greenberg, P.; Andrews, T.; Jagpal, S.; Reilly, N.; et al. Prevalence of SARS-CoV-2 infection in previously undiagnosed health care workers at the onset of the U.S. COVID-19 epidemic. medRxiv 2020. [CrossRef]

21. Houlihan, C.F.; Vora, N.; Byrne, T.; Lewer, D.; Kelly, G.; Heaney, J.; Gandhi, S.; Spyer, M.J.; Beale, R.; Cherepanov, P.; et al. Pandemic peak SARS-CoV-2 infection and seroconversion rates in London frontline health-care workers. Lancet 2020, 396, e6-e7. [CrossRef]

22. Rivett, L.; Sridhar, S.; Sparkes, D.; Routledge, M.; Jones, N.K.; Forrest, S.; Young, J.; Pereira-Dias, J.; Hamilton, W.L.; Ferris, M.; et al. Screening of healthcare workers for SARS-CoV-2 highlights the role of asymptomatic carriage in COVID-19 transmission. eLife 2020, 9, e58728. [CrossRef]

23. Shields, A.; Faustini, S.E.; Perez-Toledo, M.; Jossi, S.; Aldera, E.; Allen, J.D.; Al-Taei, S.; Backhouse, C.; Bosworth, A.; Dunbar, L.A.; et al. SARS-CoV-2 seroprevalence and asymptomatic viral carriage in healthcare workers: A cross-sectional study. Thorax 2020, 75, 1089-1094. [CrossRef]

24. Sahu, A.K.; Amrithanand, V.; Mathew, R.; Aggarwal, P.; Nayer, J.; Bhoi, S. COVID-19 in health care workers-A systematic review and meta-analysis. Am. J. Emerg. Med. 2020, 38, 1727-1731. [CrossRef] 
25. Ran, L.; Chen, X.; Wang, Y.; Wu, W.; Zhang, L.; Tan, X. Risk Factors of Healthcare Workers with Coronavirus Disease 2019: A Retrospective Cohort Study in a Designated Hospital of Wuhan in China. Clin. Infect. Dis. 2020, 71, 2218-2221. [CrossRef]

26. Iversen, K.; Bundgaard, H.; Hasselbalch, R.B.; Kristensen, J.H.; Nielsen, P.B.; Pries-Heje, M.M.; Knudsen, A.D.; Christensen, C.E.; Fogh, K.; Norsk, J.B.; et al. Risk of COVID-19 in health-care workers in Denmark: An observational cohort study. Lancet Infect. Dis. 2020, 20, 1401-1408. [CrossRef]

27. Eyre, D.W.; Lumley, S.; O’Donnell, D.; Campbell, M.; Sims, E.; Lawson, E.; Warren, F.; James, T.; Cox, S.; Howarth, A.; et al. Differential occupational risks to healthcare workers from SARS-CoV-2 observed during a prospective observational study. eLife 2020, 9, e60675. [CrossRef]

28. Lombardi, A.; Consonni, D.; Carugno, M.; Bozzi, G.; Mangioni, D.; Muscatello, A.; Castelli, V.; Palomba, E.; Cantù, A.; Ceriotti, F.; et al. Characteristics of 1573 healthcare workers who underwent nasopharyngeal swab testing for SARS-CoV-2 in Milan, Lombardy, Italy. Clin. Microbiol. Infect. 2020, 26, 1413.e9-1413.e13. [CrossRef]

29. de Sire, A.; Marotta, N.; Raimo, S.; Lippi, L.; Inzitari, M.T.; Tasselli, A.; Gimigliano, A.; Palermo, L.; Invernizzi, M.; Ammendolia, A. Psychological Distress and Work Environment Perception by Physical Therapists from Southern Italy during COVID-19 Pandemic: The C.A.L.A.B.R.I.A Study. Int. J. Environ. Res. Public Health 2021, 18, 9676. [CrossRef]

30. Farì, G.; de Sire, A.; Giorgio, V.; Rizzo, L.; Bruni, A.; Bianchi, F.P.; Zonno, A.; Pierucci, P.; Ranieri, M.; Megna, M. Impact of COVID-19 on the mental health in a cohort of Italian rehabilitation healthcare workers. J. Med. Virol. 2021, 94, 110-118. [CrossRef]

31. Calò, F.; Russo, A.; Camaioni, C.; De Pascalis, S.; Coppola, N. Burden, risk assessment, surveillance and management of SARS-CoV-2 infection in health workers: A scoping review. Infect. Dis. Poverty 2020, 9, 139. [CrossRef]

32. Lahner, E.; Dilaghi, E.; Prestigiacomo, C.; Alessio, G.; Marcellini, L.; Simmaco, M.; Santino, I.; Orsi, G.B.; Anibaldi, P.; Marcolongo, A.; et al. Prevalence of SARS-CoV-2 Infection in Health Workers (HWs) and Diagnostic Test Performance: The Experience of a Teaching Hospital in Central Italy. Int. J. Environ. Res. Public Health 2020, 17, 4417. [CrossRef]

33. Crispo, A.; Bimonte, S.; Porciello, G.; Forte, C.A.; Cuomo, G.; Montagnese, C.; Prete, M.; Grimaldi, M.; Celentano, E.; Amore, A.; et al. Strategies to evaluate outcomes in long-COVID-19 and post-COVID survivors. Infect. Agents Cancer 2021, 16, 62. [CrossRef]

34. Jin, H.; Chen, Y.; Fu, Q.; Qu, Q. Occupational risk factors of contracting COVID-19 among health workers: A systematic review. Work 2021, 69, 721-734. [CrossRef]

35. Cascella, M.; Marinangeli, F.; Vittori, A.; Scala, C.; Piccinini, M.; Braga, A.; Miceli, L.; Vellucci, R. Open Issues and Practical Suggestions for Telemedicine in Chronic Pain. Int. J. Environ. Res. Public Health 2021, 18, 12416. [CrossRef]

36. Cascella, M.; Miceli, L.; Cutugno, F.; Di Lorenzo, G.; Morabito, A.; Oriente, A.; Massazza, G.; Magni, A.; Marinangeli, F.; Cuomo, A.; et al. A Delphi Consensus Approach for the Management of Chronic Pain during and after the COVID-19 Era. Int. J. Environ. Res. Public Health 2021, 18, 13372. [CrossRef]

37. Gallè, F.; Sabella, E.A.; Roma, P.; Da Molin, G.; Diella, G.; Montagna, M.T.; Ferracuti, S.; Liguori, G.; Orsi, G.B.; Napoli, C. Acceptance of COVID-19 Vaccination in the Elderly: A Cross-Sectional Study in Southern Italy. Vaccines 2021, 9, 1222. [CrossRef] 